\title{
ORGANIZACIÓN FORESTAL COMUNAL Y SUSTENTABILIDAD EN SAN JUAN NUEVO (MICHOACÁN): PODER Y CONFLICTO EN TORNO A LOS USOS LOCALES, NACIONALES E INTERNACIONALES DE LA RECIPROCIDAD *
}

\author{
Sílvia BOFILL POCH ** \\ Dept. de Antropología Social, Universidad de Barcelona
}

ABSTRACT: National and regional peasant movements during the 1970s and the 1980s struggling to create a legal and productive space for peasant carved out by state intervention did provide a space within which communities as San Juan Nuevo could organize and regain some control over their forest resources. State policies and dramatic forestry subsequent reforms during the 90 s were deadened by implementing forest supporting programs, such as the National Reforestation Program within the National Solidarity Program, which presumed to reshape both productive and political relations in Mexico's forest dependent communities. Ultimately however, state institutions as well as international supporting agencies as the World Bank, despite the rhetorical commitment to peasant production and environmental issues, largely articulate with private industrial interests and act as mechanisms that secured control over forest resources by external interests. San Juan forestry model, preferentially supported, provides a window for understanding how industrial interests, state practices and community strategies intersect. In fact it illuminates new clientelistic strategies embedded in forest policy and neo-liberal discourse more generally, ones in direct contradiction to the solidarity's democratic, neutral international agencies and state rhetoric.

* Esta investigación es parte de la Tesis doctoral que estoy llevando a cabo sobre organización comunal forestal en San Juan Nuevo y ha sido realizada con el apoyo de una Beca Predoctoral de Formación de Profesorado Universitario otorgada por el Ministerio de Educación y Cultura español, y en el marco del Proyecto de Investigación: "La reciprocidad como recurso humano" financiado por el MEC dentro del Programa Sectorial de Promoción General del Conocimiento (PB98-1238). Los datos etnográficos utilizados, así como las voces etnográficas que aparecen entrecomilladas en el texto han sido extraídos de un extenso trabajo de campo realizado durante los años 1999 y 2000 en la comunidad en cuestión.

** E-mail: bofill\&trivium.gh.vb.es 
A lo largo de los últimos 20 años y como resultado de los movimientos campesinos iniciados en la década los 70 en México y concretamente en el Estado de Michoacán, la comunidad indígena de Nuevo San Juan Parangaricutiro, ubicada geográfica y culturalmente en la Meseta Purhépecha, ha ido consolidándose, en el marco de la silvicultura comunitaria mexicana, como una de las comunidades mejor y más eficientemente organizadas a nivel forestal del país ${ }^{1}$. Tras más de medio siglo de expropiación y sometimiento del aprovechamiento del recurso forestal comunal a los intereses del mediano y gran industrial maderero, regional y nacional, la comunidad indígena de San Juan logra, al amparo de las reformas institucionales en materia agraria y forestal del gobierno de Luís Echevarría (1970-1976), iniciar un proceso de reapropiación productiva del recurso forestal comunal. En 1977, y bajo un programa de «socioproducción" que promueve la organización del sector social (ejidos y comunidades agrarias) bajo nuevas formas asociativas y que se orienta, en el caso de la Meseta, a imponer orden y reactivar una caótica, poco integrada (horizontal y verticalmente) y todavía muy ineficiente industria maderera nacional forestal, nace la Unión de Ejidos y Comunidades Indígenas Forestales de la Meseta Tarasca Lic. Luís Echevarría Álvarez, de la que la comunidad de San Juan, junto a otros 25 núcleos agrarios, será socio fundador. La Unión posibilitará la obtención de los primeros permisos de explotación forestal, así como el desarrollo de una infraestructura productiva dirigida a asumir íntegra y conjuntamente el proceso de extracción, industrialización y comercialización del recurso forestal de sus miembros integrantes.

Pese a haber constituido durante un tiempo la experiencia organizativa forestal más grande y esperanzadora de la Meseta, la Unión no funcionó debido fundamentalmente a problemas administrativos y de corrupción interna. La experiencia, el liderazgo y cierta infraestructura básica heredada de la Unión permitirán, no obstante, a San Juan iniciar por cuenta propia un proceso independiente de organización para la producción forestal. Así, con el apoyo inicial de la planta mixta de papel y conglomerado CEPAMISA S.A. (Celulósicos y Papel de Michoacán, S.A., filial del Grupo Chihuahua) instalada en Morelia desde 1981, San Juan Nuevo logra en 1983, tras adquirir su primer aserradero, consti-

1 La comunidad indígena (o agraria) de Nuevo San Juan Parangaricutiro (aprox.7000 hab.) forma parte del municipio de Nuevo Parangaricutiro (aprox.15.000 hab) que a su vez está integrado por 4 ejidos y por pequeñas propiedades, algunas surgidas de las «Leyes de desamortización" de finales del XIX, y otras escrituradas (a costa de lo que hoy se considera terreno comunal) a partir de los años 40 y hasta los 70 en base a los sistemas productivos forestal y aguacatero. La comunidad posee más de 10.000 has forestales (pino-encino). 
tuir la empresa social forestal Aprovechamientos Forestales de la CINSJP. Su rápido crecimiento, ligado a una extraordinaria habilidad para sobrevivir económica y políticamente a los embates de la política neoliberal de los 80 y los 90 , han convertido a San Juan, tras recibir en 1986 la Concesión de los Servicios Técnicos Forestales y afianzarse como una de las empresas sociales técnicamente más desarrolladas del país, en una organización "modelo» (integral, racional y sustentable) con reconocimiento a nivel regional, nacional e internacional ${ }^{2}$. Actualmente la empresa procesa más de 60.000 metros cúbicos de madera en rollo anuales (pino, encino y otras hojosas) llegando a una utilidad neta anual que oscila entre los $\$ 6.500 .000$ y los $\$ 8.000 .000$ (nuevos pesos mexicanos), como resultado de una diversificación productiva muy amplia que abarca la producción de astilla para la fabricación de celulosa, una fábrica de muebles y molduras, una destiladora de resina, tres viveros forestales, un programa agropecuario - agrícola, pecuario y frutícola-, un programa de ecoturismo y otras actividades que proporcionan alrededor de 900 empleos directos y más de 400 indirectos 3 .

Sin duda, la perfecta articulación del proyecto empresarial con determinados intereses industriales (y políticos) regionales ha resultado crucial en la conformación y el desarrollo económico de la empresa. No obstante, el papel ejercido por sus líderes y dirigentes así como la participación e implicación activa de los comuneros en el proyecto también han resultado cruciales en un proceso que se asume como empresarial, pero fundamentalmente como social. La tarea de construir el "interés común" en torno a un proyecto de reapropiación productiva del bosque que por su mismo impulso transformador supone la confrontación de las estructuras políticas y económicas regionales pero también locales precedentes será asumida, en buena medida, por un nuevo órgano político comunitario cuyos miembros, comuneros todos ellos, se irán consolidando poco a poco, en base a un contundente liderazgo político y empresarial, como la nueva clase política dirigente de la empresa y del núcleo agrario o comunidad, en general. Así, sobre una dinámica de negociación (y renegociación) constante de dicho interés que, aunque la contenga, rebasa ampliamente una expectativa de satisfacción material (puesto que se adentra de lleno en el complejo universo social y cultural sanjuaneco) se irán definiendo un conjunto de normas disciplinarias que regulan una nueva división del trabajo, atribuyen nuevos derechos y

2 La empresa comunal obtiene en 1985 el Premio al Mérito Nacional Forestal 1984 y en 1997 la Certificación del Buen Manejo de los Recursos Forestales por parte del Consejo Mundial de Manejo Forestal (FSC).

3 Fuente: «Departamento de Contabilidad", Aprovechamientos Forestales CINSJP. 
obligaciones y legitiman determinadas prácticas de distribución de bienes y servicios, materiales y simbólicos, así como nuevas formas de intermediación y representación política, dentro y fuera de la comunidad.

El interés colectivo se verá, no obstante, ampliamente rebasado (o transgredido) en pro de un interés particular, por el grupo dirigente durante los noventa, cuyas acciones, normas, luchas y estrategias internas por mantener (o reconquistar) el poder dentro de la organización, conllevarán una generalizada crisis de legitimidad no únicamente de la dirigencia, sino del proyecto comunal en general. Lejos de analizar en profundidad este proceso me propongo apuntar brevemente el papel que las relaciones de reciprocidad, contenidas en la lógica de la negociación del «interés común», juegan en la construcción del consenso colectivo sobre el que descansa la legitimidad social y moral tanto del proyecto como de la autoridad instituida. Identificar algunos de estos procesos nos permitirá localizar, así mismo, dinámicas y espacios privilegiados de contención y explosión del conflicto dentro de la comunidad. Para ello será interesante recurrir a nociones como "justicia distributiva", "economía moral", "equidad" o "legitimidad", todas ellas teorizadas en torno a conceptos como poder, autoridad, moralidad, rebeldía u obediencia por autores como B. Moore (1996 (1978)), J. Scott (1986 (1977)) o P. Bourdieu (1991 (1980)), y más recientemente por S. Narotzky y P. Moreno (2000).

\section{Reciprocidad y organización forestal comunal}

Durante más de cuarenta años la extracción e industrialización del recurso forestal comunal en San Juan estuvo en manos fundamentalmente del mediano y gran empresario maderero, contratista o rentista. Como resultado de una práctica extractiva masiva e irracional desarrollada al margen de disposiciones técnicas y legales, en complicidad con la propia administración forestal, y por medio del intermediarismo local, económico y político (caciques), amplias áreas de San Juan y de la Meseta en general fueron ferozmente devastadas, generando una simultánea estratificación así como una desestabilización económica y social al interior de las comunidades ${ }^{4}$. El decreto de una veda forestal indefinida en $\mathrm{Mi}$ -

4 Dicha estructura de intermediación local estuvo dominada por las familias fuertes o caciques del pueblo, indígenas o mestizos (muchos de ellas en calidad de pequeños propietarios), cuyo poder y posición privilegiada se desprende directamente del control ejercido desde finales 
choacán en la década de los cincuenta, no sólo no frenó el saqueo de los bosques, sino que involucró a las comunidades en la práctica del clandestinaje como estrategia de abastecimiento a los industriales del aserrío. La proliferación, por otro lado, del taller familiar semi-empresarial a nivel local para la fabricación de caja de empaque para fruta de exportación (y otros productos artesanales de madera) en la década de los sesenta, así como la entrada masiva de "hacheros», «burreros» $\mathrm{y}$ «carretoneros» como proveedores (clandestinos) de madera para dichos talleres (muchos de ellos también en régimen de ilegalidad) aceleró los índices de deforestación y la práctica de la clandestinidad. La introducción en la región del cultivo del aguacate en la década de los setenta aceleraría este proceso y afianzaría una compleja red de interés al servicio del mediano y gran empresario frutícola y fundamentalmente aguacatero (cuyo capital proviene de la misma industria maderera), difícil de desarticular frente a una propuesta organizativa alternativa.

$\mathrm{Al}$ margen de dichas prácticas extractivas de carácter industrial, el bosque ha constituido tradicionalmente en San Juan fuente de extracción resinera y recolección individual o familiar de leña, maderas para la fabricación de tejamanil u otras utilidades para uso doméstico, complementarias de una economía maicera de subsistencia y ajenas, en cualquier caso, a la práctica comunitaria y organizada del cultivo del bosque. Expulsar a madereros y contratistas, imponer "orden" sobre una "desorganizada" extracción forestal (al servicio del empresario aguacatero), e introducir lo que se consideró una "nueva manera de ir al monte» dentro del nuevo esquema organizativo comunitario necesitó, por tanto, primeramente desarticular redes de interés económico complejas así como rearticular "hábitos» y tendencias de aprovechamiento (familiares e industriales) en torno a una nueva propuesta de centralización empresarial, técnica y administrativa del bosque, distinta totalmente a las dinámicas de extracción forestal anterior. La posibilidad de articular poseedores de monte o parceleros, hacheros, tallereros de sierra-cintas, resineros e incluso aguacateros en torno a dicha propuesta enfrenta no únicamente un reto económico básico, sino que precisa de una labor social previa de convencimiento dirigida a ganarse la confianza por un lado, y a romper con una serie de "hábitos" y prácticas políticas y sociales por otro, que contradicen o entorpecen la lógica técnica y organizativa de una nueva "cultura productiva» o silvicultura comunitaria. La separación inicial entre un liderazgo empresarial asumido por el

del XIX sobre los medios de producción (tierra y bosque) y sobre los medios de representación política, civil, agraria y tradicional. Sus intereses se han visto tradicionalmente vinculados a, y representados por, la institución eclesiástica y el clero en San Juan. Para una revisión histórica de la conformación de dichos grupos ver C. MOHENo (1986). 
Comisionado para los Aprovechamientos Forestales, responsable de dirigir económica y administrativamente el proyecto, y un liderazgo político y social encabezado por la autoridad agraria o Representante de Bienes Comunales, responsable de ir filtrando y socializando nuevos criterios organizativos que escapan al manejo propiamente empresarial constituye, en este sentido, un factor distintivo y sumamente importante del éxito de la experiencia organizativa sanjuaneca.

Sobre ambos liderazgos, empresarial y social, y sobre la práctica de una «comunalidad» empresarial que busca y negocia por distintos mecanismos el equilibrio entre el interés particular y el interés colectivo, entre lo económico y lo social, o entre la eficiencia en la toma de decisiones y la democracia participativa (propias de un práctica asamblearia), se va desdibujando una nueva forma de organización y una nueva dinámica de intercambio cuyo fin último se orienta, cuanto menos ideal y discursivamente, al incremento equitativo y generalizado del bienestar social. En este sentido, si bien la exigencia de convertir el proyecto en un negocio de naturaleza empresarial depone, en algún momento, demandas de carácter social que ceden ante imperativos innegociables de carácter productivo (precio de la madera, criterios y ritmos laborales, sueldos, manejo/reparto de utilidades, etc.), dichas demandas exigen con igual fuerza, en otros momentos, ser atendidas de acuerdo a principios o prácticas de naturaleza no empresarial «diferentes totalmente a los que contempla el negocio como negocio". Así por ejemplo, ofrecer un precio razonable o "derecho de monte» por el corte de madera a los comuneros o parceleros poseedores de monte, ofrecer un precio preferencial por la compra de madera ("cartilla de abastecimiento") a los talleres sierra-cinta, abastecidos clandestinamente a un precio muy inferior, generar empleo como factor de retención migratoria o dotar de infraestructura y servicios sociales al conjunto de la comunidad frente a una política estricta de capitalización de la empresa serán algunos de los pactos y negociaciones sobre los que ambos liderazgos, dentro de los límites y exigencias económicas ineludibles, deberán sustentar el proyecto.

Asumir desde esta nueva "cultura productiva» el control económico del bosque de forma centralizada (y corporada) a través de una empresa que se define a sí misma como social (la empresa pertenece a la comunidad) implica adquirir un sentido de responsabilidad compartida que exige, no únicamente una nueva manera de pensar y relacionarse productivamente con el bosque (asumido ahora desde una renovada idea de "bien común»), sino también con el conjunto de miembros de la comunidad: "cada equipo de trabajo no se decide de arriba para abajo sino que se decide entre ellos [...], acorde con un 
espíritu de cuerpo, un espíritu de grupo que hace que el comunero asuma las metas como propias y se esmere en dar [...], generando un ambiente de trabajo diferente al de la empresa privada, donde la sinergia del equipo es $80 \%$ superior". Esta sinergia recostada sobre la esfera de lo social se ve así mismo reforzada por una simultánea práctica de la cooperación y participación voluntarias no remuneradas directamente que permiten coordinar acciones tan importantes como el control de incendios, por medio de la creación de patrullas rotativas de vigilancia, o las campañas de poda y reforestación. La necesidad económica del crecimiento organizativo recurre en ocasiones a las faenas, actividad también voluntaria contemplada como obligación moral y practicada tradicionalmente para cubrir obras de beneficio social, y que en este nuevo contexto opera en la construcción, por ejemplo, de obras de infraestructura básica de la empresa (como el cobertizo del primer aserradero).

El rechazo a un posible manejo, inversión o distribución del dinero y del recurso (utilidades, créditos, programas gubernamentales, salarios, etc.) de acuerdo a prácticas o criterios clientelistas que pudieran desvirtuar o «abortar" el proyecto productivo y la naturaleza equitativa de la propuesta organizativa inicial queda contrapuesta, en este contexto, a la administración del negocio como negocio, de acuerdo a una gestión empresarial limpia, clara y honesta: «no se puede resolver con criterio político lo que debe resolverse con criterio empresarial». La preocupación inicial por establecer ámbitos de decisión bien diferenciados entre las esferas política y económica, es decir, entre criterios de administración política y criterios de administración propiamente empresarial (sobre los que de hecho se construye el organigrama político general de la comunidad) contiene, cuanto menos ideológicamente, el rechazo implícito a la apropiación política de un proyecto que se asume no únicamente como económico sino, fundamentalmente, como social. La mayor o menor «lucidez» de la autoridad política por ceder, negociar o delimitar su propio campo de decisión y actuación frente a lo que supone un nuevo ámbito de responsabilidad empresarial constituye y sigue constituyendo, tal y como indica uno de los líderes empresariales, un principio organizativo esencial: "[...] gran número de los procesos abortados que he visto o me ha tocado tratar han sido porque no ha habido la lucidez por parte de quien tiene el poder político para aceptar ningún criterio de tipo empresarial» 5 .

5 Estas afirmaciones anticipan o contienen a otro nivel el rechazo implícito a establecer vínculos o dinámicas de deuda, dependencia o clientelismo político o económico por ejemplo con el estado, contrarios al ideal de autosuficiencia económica, emancipación o autonomía politica por la que se aboga inicialmente. 
Este nuevo esquema organizativo presupone igualmente una conciencia política distinta tanto en relación al lugar que como dueños legítimos del recurso y del territorio los comuneros pueden y deben ocupar en la estructura económica, local y regional, en calidad de beneficiarios directos del aprovechamiento forestal, como en relación a la necesidad y conveniencia de recurrir a la práctica de la "colectividad" como premisa indispensable para dicha transformación. El recurso ideológico a la etnicidad o adscripción étnica (que opera a través de la noción de "comunidad") como motor de movilización social, y como estrategia de apropiación de las ventajas udesprendidas de la política de bienestar social de origen gubernamental» que brinda el nuevo contexto en materia indígena institucional (Vázquez 1992: 103), actúa como factor de recorporativización (en relación a la restitución agraria sobre todo) y como agente fundamental de legitimación política, social y moral en San Juan ${ }^{6}$. La gestión del interés particular pasa dentro de esta lógica, por la gestión corporada y comunitaria del recurso forestal y territorial 7 . En cualquier caso, la participación de los comuneros en las distintas áreas o esferas de trabajo de la empresa y la aceptación de determinadas normas disciplinarias, derechos y obligaciones en relación a las esferas productivas (división del trabajo y distribución de bienes y servicios) y en la toma de decisiones (autoridad), si bien parte de la idea del «interés» particular, se sustenta y legitima sobre una más amplia noción de lo que B. Moore (1996) ha denominado "justicia distributiva», en la que la equidad, contenida en esa misma idea de comunidad, aparece como principio fundamental de implicación y participación colectiva.

\section{Reciprocidad y dominación política}

La introducción por parte del nuevo órgano directivo (empresarial y comunal) de nuevos patrones de distribución y redistribución del recurso dentro del

6 Este proceso entraría dentro de lo que G. P. CASTILLE (1981) y más recientemente Luís VÁZQUEZ (1992) han calificado como un fenómeno de "repurhépechización" a partir del cual la adscripción étnica o indígena se convierte en factor estratégico de lucha política y reivindicación social. Asimismo, la comunidad agraria actual permite establecer una supuesta "continuidad" territorial y "permanencia» social históricas desde la comunidad de indios, hija de la colonización, hasta la comunidad agraria hija de la revolución (VÁZQUEZ, 1992).

Es importante constatar que uno de los objetivos fundamentales de la organización en San Juan ha sido la obtención de la Resolución Presidencial del procedimiento agrario, por medio del cual se confirma y titula jurídicamente la posesión comunal de su territorio al tiempo que se convierte en comunidad agraria «de derecho", y no únicamente «de hecho". La comunidad de San Juan la obtiene en 1991 bajo el gobierno de Salinas de Gortari. 
conjunto de la organización comunal se explica desde una búsqueda de mecanismos dirigidos a reforzar la cohesión, la participación en la empresa y su propia legitimidad en calidad de autoridad instituida. Así por ejemplo, y en atención a la "necesidad" o el "interés» codificado dentro de la lógica de reproducción social y cultural, la empresa concede créditos o préstamos preferentes puntualmente a los comuneros para cubrir necesidades económicas derivadas de un cargo religioso (dentro del sistema de cargos), de una celebración matrimonial, de un bautizo, de la organización de las fiestas patronales, u otras originadas por enfermedad, defunción, accidente, etc. La obligación de devolución establece formas específicas sostenidas de vinculación, laboral, política o moral (de larga duración) con la empresa. Bajo esta misma dinámica surgen mecanismos de premio y castigo dirigidos igualmente a cooptar y aglutinar estratégicamente determinados grupos o individuos (en Uniones productivas, por ejemplo) esenciales por sus dotes de mando, reconocimiento social, liderazgo político, etc. en la reproducción económica, política y social del proyecto empresarial. A éstos se les incentiva económicamente y/o se les otorgan espacios de interlocución privilegiada con las esferas de toma de decisiones (comunal, municipal o incluso estatal). Así mismo, el grupo dirigente busca ocupar espacios de interlocución y mediación con el estado y agentes institucionales en orden a arrancar, en la misma lógica que sugiere Scott (1986:39), recursos del exterior con los que aumentar los disponibles a distribuir, que revierten en un aumento de prestigio y reconocimiento social. De este modo, y mediante una suerte de alquimia simbólica que permite la transfiguración de relaciones de dominación económica (y de deuda) en relaciones afectivas duraderas de gratitud y reconocimiento (Bourdieu, 1980), el grupo líder dirigente garantiza, mediante el uso de vínculos de interés, la participación en la empresa, y refuerza, política y simbólicamente, la autoridad instituida.

Poco a poco se va consolidando una extensa red de interés, compromiso y lealtad que opera fundamentalmente a través de la deuda (económica o moral) y que hace uso tanto de la necesidad económica y/o del privilegio material, como de la importancia del estatus y de la búsqueda del privilegio social y moral (dentro de una todavía muy presente "economía de prestigio") como factores de participación y lealtad sostenidas. Dichos mecanismos, dirigidos estratégicamente hacia individuos o sectores neurálgicos de reproducción social, garantizan la posibilidad de acceder e «influir" vertical y piramidalmente por medio de relaciones de influencia, dependencia o fidelidad parental, vecinal o barrial, sobre las bases amplias de la sociedad. Así, las dinámicas de intercambio, ayuda mutua y reciprocidad horizontal tradicionalmente incrustadas den- 
tro de la estructura de reproducción material y social local, quedan rearticuladas en torno a nuevos patrones de distribución y reciprocidad ${ }^{8}$. De este modo, la articulación de dichos patrones en torno a principios o espacios vitales de reproducción social y moral en San Juan, entre los que el ciclo festivo cívico-religioso ocupa un lugar central, desdibuja una extensa red de influencia y movilización social que actúa no únicamente como factor de cohesión social, sino como una poderosa herramienta de control (y legitimación) social.

Las reformas neoliberales y los subsiguientes reajustes en materia comercial, agraria y forestal durante los noventa (Nueva Ley Forestal, 1992, Tratado de Libre Comercio de América del Norte, 1994, etc.) sitúan a San Juan y al sector social forestal en general frente a un nuevo reto comercial derivado de una rígida política forestal reorientada hacia el interés industrial del gran capital privado extranjero. Contrariamente a muchas de las experiencias organizativas forestales en la Meseta (como los aserraderos comunales de Tingambato, Tanaco o Cherán) San Juan Nuevo logrará esquivar o contrarrestar tales efectos (incremento de costes de producción, competitividad desleal, incremento de importaciones, etc.) por medio de la captación privilegiada de recursos institucionales canalizados por medio del "Programa Nacional de Solidaridad" puesto en marcha por el gobierno de Salinas de Gortari (PRONARE, FONAES, etc.). La proyección, por otro lado, de San Juan hacia puestos de representación institucional estatal o federal (diputaciones locales y federales, dirección de la UNOFOC, Unión Nacional de Organizaciones en Forestería) que le permiten beneficiarse y captar recursos de instancias nacionales e internacionales como el Banco Mundial se produce simultáneamente a su proyección gubernamental, dentro y fuera de la Meseta, como una de las más exitosas experiencias en forestería comunitaria del país.

Mas allá de una clara intencionalidad política (y clientelista) de dichas proyecciones (Nelson 1996), orientadas no sólo a afianzar la presencia del gran capital industrial papelero en la Meseta (CEPAMISA) sino a reconquistar espacios perdidos

8 Estas relaciones verticales propias de sistemas de dominación y dependencia de carácter "clientelar" (o caciquil) y su correlación respecto a la concetración del control sobre el recurso productivo (en los mandos de la empresa) respondería a una dinámica similar a la que M. CALDERÓN (1994: 47) señala para calificar los clientelismos en general: «El monopolio de recursos escasos es un contexto adecuado para la creación de relaciones clientelares. Las posibilidades de encontrar satisfacción se encuentran más en la vinculacion con el patrón (cacique) que con aquellos sujetos que experimentan las mismas condiciones de existencia. Así, se constituye un orden en donde los lazos de solidaridad se extienden verticalmente en detrimento de relaciones horizontales, consolidándose una estructura social ordenada jerárquicamente» (el subrayado es mío). 
de una truncada legitimidad política y social del Partido Revolucionario Institucional (PRI) (agudizada desde la entrada de la oposición en la arena política nacional en 1988 de la mano del Partido de la Revolución Democrática [PRD]) ${ }^{9}$, una cada vez más compleja y comprometida ubicación de San Juan en el panorama políico y económico regional (siempre desde su adscripción al PRI), se traduce en una creciente centralización en la toma de decisiones y una intensificación de los mecanismos internos de control y contención de la "desviación" social. Así por ejemplo, una cada vez menos equitativa y transparente administración del recurso, nuevas formas de redistribución de utilidades, créditos o ayudas de programas gubernamentales, la acentuación de las jerarquías y diferencias económicas internas, una distribución desigual del dinero, sueldos alta e inexplicablemente diferenciados, práctica de la ostentación desmedida y del secretismo interno, poca transparencia en el manejo de las cuentas, precios injustificadamente bajos pagados al comuneroparcelero por su madera ${ }^{10}$, o una radicalización de los conflictos internos por la tenencia de la tierra, entre otros, acrecientan las tensiones y el conflicto derivados de un proceso de profunda diferenciación interna. El desvinculamiento moral de un proyecto que deja de percibirse como propio por parte de un número creciente de comuneros se correlaciona con una intensificación de las medidas coactivas o coercitivas ${ }^{11}$ en orden no únicamente a contener la disidencia, sino a encubrir las diferencias of facciones internas y absorber posibles desviaciones coyunturalmente surgidas en pugnas por la confrontación o el reposicionamiento personal en la estructura de poder ${ }^{12}$. El castigo a la disidencia política por ejemplo (que en sí misma es expresión de conflictos que se manifiestan a otro nivel y en los que ahora no podemos entrar), la contribución a la financiación de las campañas políticas es-

9 Recordemos que Michoacán, y especialmente la Meseta, son cuna del "neocardenismo" como movimiento político opositor y que la Meseta es escenario de movimientos indígenas contestatarios afines ideológicamente al movimiento zapatista (DIETZ, 1999). San Juan representa no únicamente el bastión priista de una región (y de un Estado como Michoacán) profundamente perredistas, sino que su éxito organizativo empresarial da muestra tal y como señala un comunero de San Juan de "la lucha por la permanencia indígena sin necesidad de estar en una resistencia».

${ }_{10} \mathrm{El}$ precio de la madera de pino en rollo, por ejemplo, se paga al comunero-poseedor a 136 pesos $/ \mathrm{m} 3$ mientras que el precio que ofrecen compradores externos a particulares oscila entre los 450 y los 500 pesos $/ \mathrm{m}^{3}$.

1 Algunos de los mecanismos más frecuentes de castigo son el corte de suministro de agua para huertas, despido del trabajo (personal o de familiares), expulsión del censo comunal, suspensión temporal de sueldo o supresión de apoyos canalizados desde el estado.

12 Podríamos aplicar un razonamiento similar al que J. SCOTT (1986: 59) realiza para explicar las dinámicas generales del patronazgo: "[...] cuando no existen fuertes garantías externas que protejan la riqueza y el rango, la posición de las élites locales depende en último término de la cantidad de gente que puedan reunir para un enfrentamiento". 
tatales del PRI y la imposición del voto de forma obligada y corporada como expresión fundamental e innegociable de lealtad al "Partido" que los alimenta y a "La Comunidad» (convertida ya en sinónimo del grupo dirigente), fomentan un creciente cuestionamiento (o crisis de legitimidad) de los mecanismos de elección, las formas de representación y la autoridad instituidos.

Estos mecanismos, convertidos en parte estructural del engranaje político y económico de dicha clase dirigente y por ello mismo en el obstáculo más fuerte de cualquier propuesta política alternativa, afloraran con fuerza frente a la amenaza de continuidad que supone una posible confrontación del orden social instituido por los sectores más disconformes, agudizada por otro lado frente a una inminente caída del Sistema (PRI), refugio y espejo político de la Comunidad 13. Poco a poco el «espíritu de la Solidaridad» vendría a reemplazar el «espíritu comunitario" de manera que, tal y como expresa un comunero, programas como el PRONASOL lejos de contribuir al desarrollo económico y social de la empresa (pese a canalizar una gran cantidad de apoyo económico) «sembraron la corrupción y fomentaron la deslealtad, de modo que aquel primer esfuerzo por crear un nuevo marco de responsabilidad colectiva y obtener logros económicos propios, cede ante el clientelismo y la compra de incondicionales; el talento, la disciplina y la creatividad pasan ahora a segundo término". Los reclamos de autosuficiencia económica y autonomía política respecto a un estado que "ha convertido a San Juan en un apéndice del PRI" se convierten, en este contexto, en los ejes centrales de crítica y cuestionamiento del Partido, de la clase política dirigente en San Juan y del proyecto comunitario en su totalidad: "[...] al aceptar ese dinero de riesgo del gobierno hemos vendido parte de nuestra propia estima. Esto desvirtúa absolutamente el movimiento genuino de años $\mathrm{y}$ años de ser autosuficientes y no estar dependiendo de nadie».

\section{A modo de conclusión}

Una distribución injustificadamente «desigual» de la riqueza, un excesivo autoritarismo en la toma de decisiones y un rechazo generalizado a los nuevos criterios de inclusión/exclusión impuestos «arbitrariamente» por la dirigencia,

13 Es importante remarcar que esta profunda crisis de legitimidad experimentada por la clase política de San Juan coincide con la agudización de la crisis política sufrida por el Partido Oficial que conducirá a la derrota electoral el 2 de julio de 2000 y al ascenso a la Presidencia de la República del candidato del Partido de Acción Nacional, Vicente Fox. 
que confrontan y atraviesan sentimientos de pertenencia y de relación social profundamente enraizados en la historia vivida de la gente de San Juan, forjarán la fisionomía del proyecto comunitario hasta enero del 2001, fecha en que el candidato de la clase política de la Comunidad es derrotado en los comicios a Comisariado de Bienes Comunales (autoridad agraria) por un candidato opositor que logra aglutinar a un sector amplio disconforme, crítico y mayormente desplazado al interior de la comunidad ${ }^{14}$. Dicha ruptura presupone el agotamiento de un esquema de liderazgo que, tras haber conducido ininterrumpidamente el proyecto comunitario a lo largo de dos décadas, comienza a ser percibido e identificado por el grueso de los actores como un nuevo cacicazgo.

Un primer análisis de esta primera confrontación política interna del proyecto comunitario (que aunque leve y todavía incipiente prefigura nuevas formas de negociación política intracomunal, municipal y estatal) y de los cada vez más «inequitativos" patrones de reciprocidad, codificados social y moralmente en el nuevo marco organizativo, nos advierte de la complejidad de un proceso de transformación que, pese a su cometido reivindicativo y de confrontación de la desigualdad, económica, política, social y cultural se explica desde la reproducción (o reubicación) de los mecanismos de distribución y apropiación desigual de la riqueza, el poder y el prestigio. La naturaleza de dichos mecanismos más que una ruptura, y pese a su vocación corporativa, prefigura el reacomodo histórico de la lucha de clases en San Juan. $\mathrm{Y}$ es precisamente en dicha encrucijada donde se desdibujan las «potencialidades disruptivas" de la noción de reciprocidad (Narotzky y Moreno, 2000).

\section{Referencias bibliográficas}

BOURDIEU, P. 1991 (1980): El sentido práctico, Madrid: Taurus.

CALDERÓN, M. (1994): Violencia politica y elecciones municipales, Zamora/México D.F:: COLMICH/Instituto Mora.

14 El grupo crítico intracomunitario ha encontrado junto con el sector de la pequeña propiedad confrontado por la Comunidad expresión política opositora en el Partido de la Revolución Democrática (PRD). A través de él ha canalizado el conflicto y articulado la lucha por la Presidencia Municipal. 
CASTILLE, G. P. (1981): "On the Tarascanness of the Tarascans and the Indianess of the Indians», en Persistent Peoples. Cultural Enclaves in Perspective, Tucson: University of Arizona Press.

DiETz, G. (1999): La comunidad purhépecha es nuestra fuerza, Quito: Abya-Yala.

Moheno, C. (1986): Las historias y los hombres de San Juan, Zamora/México D.F.: COLMICH-CONACYT.

MOORE, B. 1996 (1978): La injusticia: bases sociales de la obediencia y la rebelión, México D.F.: UNAM.

NAROTZKY, S., y MORENO, P. (2000): «La reciprocidad olvidada: reciprocidad negativa, moralidad y reproducción social», en Hispania. Revista Española de Historia, CSIC, vol. LX/I, n. ${ }^{\circ} 204$, pp. 127-160.

NELSON, L. (1996): Neoliberalism as contested ideological terrain: state practices and pesasant agencies in Michoacán, Mexico, Tesis presentada para el Master of Arts, University of Washington.

SCOTT, J. 1986 (1977): «¿Patronazgo o explotación?», en E. Gellner y otros, Patronos $y$ clientes, Barcelona: Júcar, pp. 35-61.

VAZQUEZ, L. (1992): Ser indio otra vez. La purhepechización de los tarascos serranos, México D.F.: Consejo Nacional para la Cultura y las Artes. 